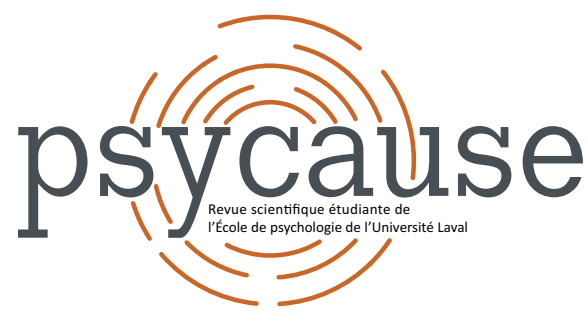

\title{
PSYCAUSE
}

Revue scientifique étudiante de l'École de psychologie de l'Université Laval

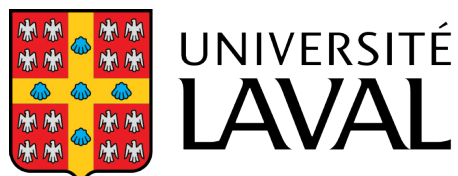

Faculté des sciences sociales École de psychologie

NOVEMBRE 2020 - VOL. $10 \mathrm{~N}^{\circ} 2$

\section{LE RAPPEL DE RÊVES CHEZ LES Â̂NÉS PRÉSENTANT UN TROUBLE COGNITIF LÉGER}

Florence BELZILE', Geneviève SANFAÇON-GAGNON¹, Béatrice SASSEVILLE1',

Mélissa MAILLOUX1 \& Célyne BASTIEN²

${ }^{1}$ École de Psychologie, Université Laval,

${ }^{2}$ Centre de recherche CERVO, Québec, Canada

*florence.belzile-marsolais.1@ulaval.ca

\section{Pour citer l'article}

Belzile, F., Sanfaçon-Gagnon, G., Sasseville, B., Mailloux, M., \& Bastien, C. (2020). Le rappel de rêves chez les aînés présentant un trouble cognitif léger. Psycause: Revue scientifique étudiante de l'École de psychologie de l'Université Laval, 10(2), 17-18. 
Savard, J., Villa, J., Ivers, H., Simard, S., \& Morin, C. M. (2009). Prevalence, natural course, and risk factors of insomnia comorbid with cancer over a 2-month period. Journal of Clinical Oncology, 27(31), 5233-5239. https://doi. org/10.1200/JCO.2008.21.6333

Sirois, F. M. et Molnar, D. S. (2016). Perfectionnism, Health and Well-Being. London: Springer.
Société canadienne du cancer. (2020). Vue d'ensemble des statistiques sur le cancer. Repéré à https://www.cancer.ca/ fr-ca/cancer-information/cancer-101/cancer-statistics-at-aglance $/$ ?region $=q c$

White, C. \& Schweitzer, R. (2000). The role of personality in the development and perpetuation of chronic fatigue syndrome. Journal of Psychosomatic Research, 48(6), 515524. https://doi.org/10.1016/s0022-3999(00)00087-8

\title{
Pour citer l'article
}

Séguin-Kaercher, N., Cadorette, J., Massicote, V., Ivers, H., \& Savard, J. (2020). La relation entre le perfectionnisme et les symptômes somatiques chez les femmes atteintes d'un cancer du sein. Psycause: Revue scientifique étudiante de l'École de psychologie de l'Université Laval, 10(2), 15-17.

\section{LE RAPPEL DE RÊVES CHEZ LES AÎNÉS PRÉSENTANT UN TROUBLE COGNITIF LÉGER}

\author{
Florence BELZILE¹, Geneviève SANFAÇON-GAGNON¹, Béatrice SASSEVILLE¹, Mélissa MAILLOUX \& Célyne BASTIEN² \\ ${ }^{1}$ École de Psychologie, Université Laval, ${ }^{2}$ Centre de recherche CERVO, Québec, Canada \\ *florence.belzile-marsolais.1@ulaval.ca
}

\section{Mots-clés : Rappel de rêves, sommeil, trouble cognitif léger, maladie d'Alzheimer}

Chez les aînés, la qualité du sommeil diminue en raison d'une réduction normale et progressive de la durée du temps passé dans le stade du sommeil paradoxal (SP), soit le stade associé à une fréquence de rappel de rêves (FRR) plus élevée. En ce sens, les écrits scientifiques démontrent qu'avec le vieillissement, il existe une diminution de la FRR (Guénolé, Marcaggi, Baleyte, \& Guarma, 2010). Comparativement au vieillissement normal, les individus ayant un trouble cognitif léger de type amnésique (TCL-a) présentent une réduction encore plus marquée du temps passé en SP. Ce trouble, prodrome probable delamaladied'Alzheimer(MA), secaractérise par des difficultés objectives et subjectives de mémoire (Albert et coll., 2011). Avant même l'apparition de déficits cognitifs, un sommeil de mauvaise qualité est susceptible de prédire le développement du TCL-a (Maestri et coll., 2015). Il existe également une relation négative significative entre les déficits mnésiques et la qualité du sommeil chez cette population (Sun et coll., 2016). Actuellement, un manque d'interventions pouvant prévenir l'évolution de ce trouble vers la MA persiste. L'activité physique semble cependant associée à un risque réduit de déclin cognitif pathologique et de développement de la MA (Gasquoine, 2018). Par ailleurs, un programme d'activité physique (PAP) de 20 semaines fut efficace pour améliorer à la fois les fonctions cognitives et la qualité du sommeil des personnes atteintes d'un TCL-a y ayant participé (Bademli, Lok, Canbaz, \& Lok, 2018).
La relation entre les déficits mnésiques et la FRR semble être bien établie dans les écrits scientifiques. II serait alors essentiel d'investiguer si la FRR peut constituer un marqueur de la mémoire susceptible de prédire le déclin cognitif des personnes présentant un TCL-a. II apparaît d'autant plus important d'évaluer l'efficacité potentielle d'un PAP pour augmenter leurs capacités mnésiques, plus spécifiquement leur FRR. Ainsi, le premier objectif est d'investiguer l'efficacité d'un PAP en mesurant son influence sur la FRR des TCL-a et le second objectif est d'étudier l'efficacité d'un PAP en mesurant son influence sur les symptômes d'insomnie.

\section{Méthode}

Quinze individus de 60 ans et plus $(M=74, E T=6,67)$, dont 9 femmes, sont recrutés sur la base de la présence d'un diagnostic de TCL-a (Albert et coll., 2011). Ceux-ci doivent également présenter des difficultés subjectives de sommeil, sans nécessairement satisfaire les critères diagnostiques de I'insomnie, et un niveau faible à modéré d'activité physique au Questionnaire d'activités physiques pour les personnes âgées. Les participants ont complété: 1) le Questionnaire sur la Fréquence de Rappel de Rêves (QFRR), permettant d'évaluer la FRR; et 2) I'Index de Sévérité de I'Insomnie (ISI), évaluant les symptômes subjectifs de l'insomnie. Ensuite, le PAP de 12 semaines a débuté à raison de trois séances hebdomadaires de 60 à 90 minutes. Le QFRR et I'ISI ont été administrés une seconde fois lorsque le PAP fut achevé. 


\section{Résultats et discussion}

\section{Objectif 1}

Tel qu'attendu, les résultats révèlent que la FRR a significativement augmenté à la suite de la participation au PAP, $t 14=1,774, d=0,59$. Ces résultats concordent avec les écrits scientifiques et mettent en lumière l'efficacité du PAP pour améliorer les capacités mnésiques des personnes atteintes d'un TCL-a (Bademli et coll., 2018). En effet, le temps passé en SP se voit diminué chez ces dernières, au même titre que la FRR (Guénolé et coll., 2010). Considérant le lien entre ces deux variables, ces résultats appuient l'idée que la participation au PAP permet une augmentation du temps passé en SP, laquelle contribue à améliorer la FRR.

\section{Objectif 2}

Les résultats infirment notre hypothèse: la fréquence des symptômes d'insomnie ne diffère pas significativement selon le temps de mesure, soit avant et après la participation au PAP, $\times 2(1, N=15)=1,759, \Phi c=0,342$. La progression des déficits mnésiques, conséquente à l'évolution du trouble, s'accompagne d'une intensification des difficultés de sommeil (Sun et coll., 2016). Il est donc possible qu'à ce stade du trouble, les personnes ayant un TCL-a souffrent de difficultés de sommeil trop importantes pour qu'un PAP soit efficace pour les diminuer. Toutefois, I'indice de force d'association du Phi de Cramér indique un lien positif modéré entre les symptômes d'insomnie et le PAP. Cela suggère que l'activité physique exerce un certain effet sur la qualité du sommeil des personnes atteintes d'un TCL-a.

La présente étude est l'une des premières à mesurer l'efficacité d'un PAP sur les capacités mnésiques des personnes ayant un TCL-a. En réponse à l'absence de traitement efficace pour ralentir le déclin cognitif de cette population, elle meten évidence les bienfaits potentiels d'une intervention d'activité physique pour prévenir l'évolution de ce trouble vers la MA.

\section{Références}

Albert, M. S., DeKosky, S. T., Dickson, D., Dubois, B., Feldman, H. H., Fox, N. C., ... \& Phelps, C. H. (2011). The diagnosis of mild cognitive impairment due to Alzheimer's disease: Recommendations from the National Institute on AgingAlzheimer's Association workgroups on diagnostic guidelines for Alzheimer's disease. Alzheimer's \& Dementia, 7(3), 270279. http://doi.org/10.1016/j.jalz.2011.03.008

Bademli, K., Lok, N., Canbaz, M., \& Lok, S. (2018). Effects of physical activity program on cognitive function and sleep quality in elderly with mild cognitive impairment: A randomized controlled trial. Perspectives in Psychiatric Care, 55(3), 401-408. http://doi.org/10.1111/ppc.12324

Gasquoine, P. G. (2018). Effects of physical activity on delayed memory measures in randomized controlled trials with nonclinical older, mild cognitive impairment, and dementia participants. Journal of Clinical and Experimental Neuropsychology, 40(9). http://doi.org/10.1080/13803395 .2018.1442815

Guénolé, F., Marcaggi, G., Baleyte, J. F., \& Garma, L. (2010). Le rêve au cours du vieillissement normal et pathologique. Psychologie et Neuropsychiatrie du Vieillissement, 8(2), 87-96. http://doi.org/10.1684/pnv.2010.0209

Maestri, M., Carnicelli, L., Tognoni, G., Di Coscio, E., Giorgi, F. S., Volpi, L., ... \& Bonanni, E. (2015). Non-rapid eye movement sleep instability in mild cognitive impairment: a pilot study. Sleep medicine, 16(9), 1139-1145. http://doi. org/10.1016/j.sleep.2015.04.027

Sun, Q., Luo, L., Ren, H., Wei, C., Xing, M., Cheng, Y., \& Zhang, N. (2016). Semantic clustering and sleep in patients with amnestic mild cognitive impairment or with vascular cognitive impairment-no dementia. International Psychogeriatrics, 28(9), 1493-1502. http://doi.org/10.1017/ S1041610216000739

\section{Pour citer l'article}

Belzile, F., Sanfaçon-Gagnon, G., Sasseville, B., Mailloux, M., \& Bastien, C. (2020). Le rappel de rêves chez les aînés présentant un trouble cognitif léger. Psycause: Revue scientifique étudiante de l'École de psychologie de l'Université Laval, 10(2), 17-18. 